Editorial

\title{
El desafío de integrar teoría, investigación y práctica clínica en psicoterapia
}

\author{
The Challenge of Integrating Theory, Research and Clinical \\ Practice in Psychotherapy
}

\author{
Daniel Espinosa-Duque $\underline{\text { ORCID }}$, Mariane Krause $\underline{\text { ORCID }}$ \\ ${ }^{12}$ Instituto Milenio para la Investigación de la Depresión y la Personalidad (MIDAP), Chile. \\ 1 Universidad CES, Colombia \\ ${ }^{2}$ Pontificia Universidad Católica de Chile
}

Forma de citar:

Espinosa-Duque, D., \& Krause, M. (2020). El desafío de integrar teoría, investigación y práctica clínica en psicoterapia. Rev. CES Psico, 13(3), i-ix.

Open access

(c) Copyright

Licencia creative commons

Etica de publicaciones

Revisión por pares

Gestión por Open Journal System

DOl: http://dx.doi.org/10.21615/

cesp.13.3.15

ISSN: 2011-3080

Sobre los autores:

1. Doctor en Psicoterapia.

Docente Investigador Universidad

CES. Instituto Milenio para la

Investigación en Depresión y

Personalidad, MIDAP. Presidente

Capítulo Latinoamericano Society

for Psychotherapy Research.

Editor invitado número especial de Investigación en Psicoterapia de la Revista CES Psicología.

\section{Comparte}

f日G.
En psicoterapia, así como en otros ejercicios profesionales, hay tres elementos en juego: teoría, práctica e investigación, que se espera que evolucionen y se nutran mutuamente. Sin embargo, en el desarrollo de la psicoterapia actual, diferentes condiciones históricas y profesionales han generado importantes distanciamientos entre los estudios científicos en el área, las teorías y enfoques teóricos que dan base a la formación psicoterapéutica, y los espacios clínicos de los profesionales que ejercen dicha actividad (Von Bergen \& Krause, 2016; Watkins, 1997).

Estos distanciamientos se puede explicar por diferentes factores: 1) el predominio histórico de los modelos de formación centrados en enfoques teóricos más que en hallazgos de investigaciones empíricas; 2) los sentimientos de desconfianza que tienen algunos clínicos sobre la necesidad y utilidad de los procedimientos investigativos; 3 ) la sensación de amenaza que genera en los psicoterapeutas algunas generalizaciones reduccionistas derivadas de las investigaciones; 4) la sensación de incompatibilidad entre lo que ocurre en la psicoterapia y los métodos investigativos; 5) la escasez de motivación y de espacios de formación en los cuales los clínicos puedan apropiarse y traducir los hallazgos científicos; y 6) la creencia de que las prácticas clínicas reflexivas pueden sustituir la investigación científica, porque son similares a los procedimientos de validación y comprensión de ésta (Jiménez, 1999; Krause, 2011b).

Estos distanciamientos son un fenómeno mundial, pero son especialmente agudos en países con menor tradición científica y cuyas regulaciones en salud mental dan menos importancia a la validación científica de los servicios, como es el caso de algunos países latinoamericanos. Pese a estas limitaciones, aquellos que se interesan en la investigación en psicoterapia en esta región, suelen mantener estrechos lazos con la actividad clínica (Fernández-Álvarez \& Castonguay, 2018). Aun así, es necesario trabajar arduamente en reducir esta brecha para hacer más sólida la práctica clínica y mejorar los niveles de efectividad e impacto de la psicoterapia (Fernández-Álvarez et al., 2020). Además, el fortalecimiento del diálogo con la 
2. PhD. en Psicología de la Universidad Libre de Berlín. Profesora Titular de la Pontificia Universidad Católica de Chile. Investigadora Asociada Instituto Milenio para la Investigación en Depresión y Personalidad, MIDAP. Past-President Society for Psychotherapy Research (SPR). Editora invitado número especial de Investigación en Psicoterapia de la Revista CES Psicología. investigación abre las puertas a una implementación contextual de la psicoterapia (Wampold, 2013), que considere de manera crítica y pragmática los momentos históricos y ambientes socio-geográficos en los que se realiza.

Teniendo en cuenta lo anterior, numerosos autores han señalado la importancia de acercar los procesos de formación, las prácticas clínicas en contextos específicos y los espacios investigativos en el campo de la psicoterapia (Castonguay, Youn, Xiao, Muran, \& Barber, 2014; Castonguay \& Muran, 2015), con el fin de enriquecer y fortalecer estos tres elementos.

Hasta el momento se han utilizado y desarrollado numerosos métodos y diseños de investigación para dar respuesta a las inquietudes fundamentales y emergentes de la investigación en psicoterapia (Gelo, Pritz, \& Rieken, 2015). El diseño que abrió las puertas a numerosas hipótesis y teorías clínicas fue el estudio de caso, mediante el cual se realizan análisis sistemáticos de lo que ocurre en el espacio terapéutico a nivel descriptivo y exploratorio (Braakmann, 2014). No obstante, los ensayos clínicos articulados con la tradición médica han sido fundamentales para dar un lugar a los tratamientos psicoterapéuticos dentro de los espacios sanitarios, mostrando su efectividad, eficacia y algunos de los factores de cambio que pueden atribuírseles (Comer \& Kendall, 2013).

Estos diseños, y otros como los transversales, longitudinales, retrospectivos, de construcción y validación de instrumentos, evaluación de intervenciones, revisiones sistemáticas y meta-análisis (Gelo et al., 2015), han permitido comprender múltiples dimensiones de los procesos y resultados terapéuticos. Por su parte, los métodos de análisis cualitativos, cuantitativos y mixtos han permitido indagar desde múltiples perspectivas (pacientes, terapeutas, u observadores) los elementos que influyen en dichos procesos y resultados.

A fin de visualizar los aportes que la investigación en psicoterapia puede ofrecer a la teoría, la práctica clínica y la formación, a continuación, se revisarán sus tópicos centrales, ejemplificados con algunos hallazgos emblemáticos.

\section{Objetivos y tópicos de la investigación en psicoterapia}

En términos generales, el objetivo de la investigación en psicoterapia es responder preguntas sobre los efectos de los procedimientos psicoterapéuticos y los procesos de cambio. Una excelente propuesta ordenadora de la investigación en psicoterapia es el "modelo genérico" de David Orlinsky, que establece condiciones de entrada, incluyendo el contexto, variables de proceso y resultados en psicoterapia.

Las "condiciones de entrada" consideran las características personales con las que inician la psicoterapia los pacientes y los terapeutas, su contexto social y cultural, y las características del sistema asistencial. En cuanto a las condiciones del paciente, algunos estudios resaltan la relevancia de sus expectativas (Meyerhoff \& Rohan, 2016), su disposición a comprometerse con el terapeuta (Krause, 2011a) y de constituir una buena alianza terapéutica. Según una larga y nutritiva tradición de investigación, la alianza terapéutica establecida al inicio de la psicoterapia es el predictor más robusto de la evolución de ésta y de sus resultados (Horvath \& Greenberg, 1986). 


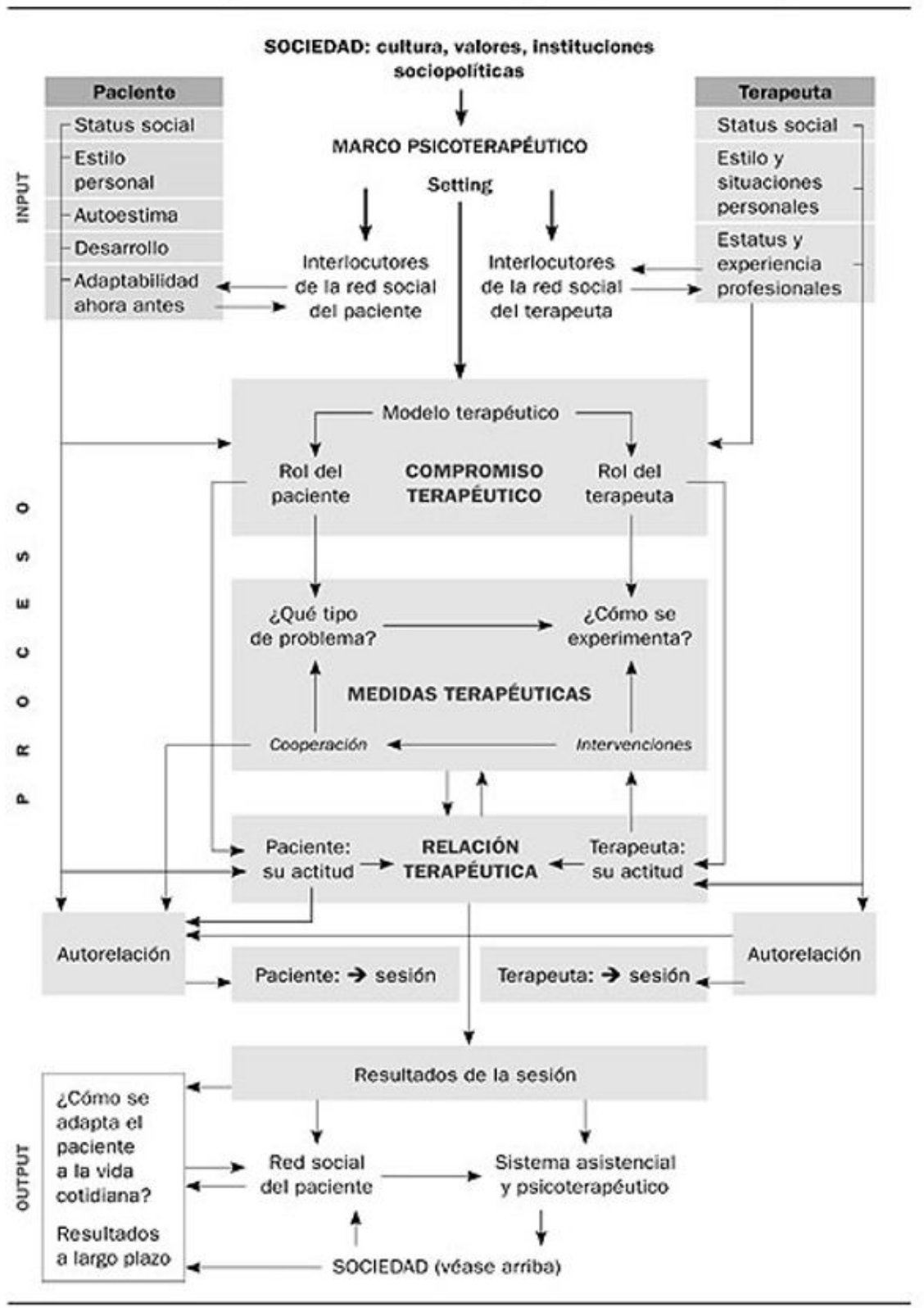

Figura 1. Modelo générico de la psicoterapia, de D. Orlinsky

Fuente: Orlinsky, D. (1994). Process and outcome in psychotherapy. En Bergin, AE., \& Garfield, SL. (eds), Handbook of psychotherapy and behavior change, Nueva York: John Wiley. Versión traducida al español tomada de Josep Solé Puig, J. (2007). Psicopatología de la adicción. Rev. Informaciones Psiquiátricas, 189. Disponible en http://www.informacionespsiquiatricas. com/anteriores/info_2007/03_189_08.htm

Los resultados de estudios sobre la influencia del contexto social y cultural muestran que la distancia cultural entre pacientes y terapeutas puede constituir una barrera al inicio de una psicoterapia (Krause, Espinosa, Tomicic, Córdoba, \& Vásquez, 2018). El contexto cultural, asimismo, conlleva una mayor o menor aceptación de los problemas psicológicos, y en ocasiones la estigmatización de éstos, lo que se constituye en un obstáculo potencial para comenzar una psicoterapia (Krause et al., 2018). Por su parte, las condiciones del sistema asistencial también influyen en aspectos estructurales de la relación de ayuda, tales como la frecuencia y duración de las intervenciones, la estabilidad o la rotación de los profesionales, entre otros (De la Parra et al., 2018; Jaramillo et al., 2018). 
Una vez instalado el marco psicoterapéutico, con las condiciones mencionadas, un particular desafío para que la terapia progrese es asegurar una definición compartida del problema, llegar a un acuerdo en las tareas que se llevan a cabo y establecer una buena relación terapéutica (Bordin, 1976; Norcross \& Lambert, 2011).

Además de estudiar la efectividad de la psicoterapia, uno de los principales intereses de los estudios actuales es comprender e identificar el "contenido de las sesiones [...] y los mecanismos a través de los cuales se logra el cambio del consultante, tanto en las sesiones, como a lo largo del tiempo" (Llewelyn \& Hardy, 2001, p. 2). Específicamente, este tipo de investigación orientada al proceso busca: (a) comprender los mecanismos del proceso de tratamiento y el proceso de cambio del cliente; (b) comprender qué aspectos del tratamiento son más importantes para el cambio; (c) contribuir al desarrollo de teorías; y (d) aportar a la formación eficaz de psicoterapeutas (Hardy \& Llewelyn, 2015).

De acuerdo con su "unidad de análisis" (Elliott, 1991), las investigaciones sobre el proceso terapéutico se pueden agrupar en estudios de micro-proceso, enfocados en sesiones, segmentos o eventos específicos (Krause \& Altimir, 2016), y estudios de macro-proceso, que abordan la terapia completa.

Los estudios de micro-proceso corresponden al segundo bloque en el diagrama de Orlinsky. Un ejemplo emblemático de este tipo de investigación es el estudio de episodios relevantes, en el que se identifican instantes específicos de las sesiones terapéuticas, que constituyen momentos de inflexión en su evolución; entre los que se destacan: episodios de cambio (Bastine, Fiedler, \& Kommer, 1989; Elliott, 1985), episodios de experiencia emocional (Angus \& Kagan, 2013), episodios de empoderamiento (Timulák \& Lietaer, 2001), momentos innovativos (Matos, Santos, Gonçalves, \& Martins, 2009), episodios de estancamiento (Herrera Salinas et al., 2009) y de ruptura de la alianza terapéutica (Safran \& Muran, 2000).

De los distintos tipos de episodios, los más estudiados son los de cambio, en los cuales, se producen "saltos" positivos en la evolución del paciente; y los de ruptura, caracterizados por quiebres temporales en la alianza terapéutica. En los primeros, los resultados de investigación destacan la importancia de la construcción de nuevos significados, y en los segundos, revelan que su reparación exitosa, lograda a través de intervenciones específicas del terapeuta, se relaciona positivamente con el cambio subsecuente; no siendo las rupturas en sí mismas, ni su frecuencia, perjudiciales para la psicoterapia (Safran, Muran, \& Eubanks-Carter, 2011; Holmqvist Larsson, Falkenström, Andersson, \& Holmqvist, 2018).

En los estudios de macro-proceso el énfasis de la investigación está puesto en la evolución del cambio a lo largo de la terapia y en la relación del proceso con el resultado final (véase el tercer bloque en el esquema de Orlinsky). El interés por la evolución del cambio ha llevado a la construcción de modelos de fases del cambio, tales como, el de Asimilación de Experiencias Problemáticas (APES, por su sigla in inglés de Assimilation of Problematic Experiences Scale) de Stiles (2002), el Modelo Transteórico de Prochaska (Prochaska \& Norcross, 2002), el Modelo Semiótico de dos fases (-TSSM, por su sigla in inglés de Two Stage Semiotic Model) (Gennaro et al., 2010) y el Modelo de Indicadores de Cambio Genéricos (Krause, Altimir, Pérez, \& De la Parra, 2015). Todos estos modelos postulan una progresión gradual del cambio, proceso que incluye cambios más básicos en etapas iniciales, que van evolucionando 
hacia cambios de mayor complejidad; además, tienen en común una aproximación trans-teórica, vale decir, muestran que las fases de la terapia son válidas para distintos enfoques psicoterapéuticos.

Los resultados de los estudios de macro-proceso develan que la evolución del cambio es un proceso irregular pero progresivo, que predice la adherencia al tratamiento y los resultados finales de la psicoterapia (Krause et al., 2015). Los principales cambios en la evolución de la terapia son representacionales, plasmados en la construcción de nuevos significados, y conllevan la integración psicológica progresiva (Stiles, 2001).

\section{El desafío de integrar teoría, investigación y práctica clínica}

Como se ha señalado anteriormente, generar espacios de integración entre los hallazgos científicos, los espacios clínicos y las teorías psicoterapéuticas es una tarea compleja, pero necesaria en la actualidad por diferentes razones que se desarrollarán a continuación. La premisa que sostiene la argumentación es que tanto la teoría, como la práctica clínica y la investigación, requieren de permanente desarrollo y actualización, cuestión que se ve favorecida cuando los tres ámbitos interactúan y se nutren mutuamente en los espacios de intervención y formación.

En primer lugar, la investigación y reflexión científica continuas permiten la evolución y optimización de las teorías que se desarrollan en un campo de conocimiento. Es claro que las primeras construcciones conceptuales de la psicoterapia, que hoy conforman la base teórica fundamental de los enfoques de intervención, nacieron de la sistematización y conceptualización de casos (Braakmann, 2014). Sería un error que los modelos de intervención, los centros de entrenamiento y los profesionales de la psicoterapia, asumieran como terminado el cuerpo de conocimiento de dicho campo, porque un mundo en continuo cambio demanda también de las teorías y las intervenciones una permanente evolución. Su progreso se nutre de los desafíos que provienen del mundo clínico, que establece las preguntas pertinentes y, posteriormente, de la investigación que implementa dichas cuestiones en diseños que pueden establecer una razonable validez para las respuestas.

En esta misma dirección, la práctica científica permite de manera rutinaria evaluar los procesos de implementación de intervenciones en diferentes contextos y culturas. Es ingenuo, en este sentido, suponer que la evidencia científica recolectada ampliamente en países desarrollados pueda ser homologable de manera directa en todos los contextos, entre ellos los latinoamericanos, sin considerar las amplias diferencias que hay a nivel psicológico y social. En consecuencia, no solo es necesario buscar la integración de la investigación, la teoría y la práctica, sino también su diseminación para ampliar la comprensión de cómo funciona la psicoterapia en diversos contextos y momentos de la historia (Fernández-Álvarez et al., 2020).

Por su parte, la investigación, si estuviese divorciada de la teoría, fácilmente podría derivar en resultados que no aporten a la acumulación de conocimientos de la disciplina. Es así como, con frecuencia, se encuentran islas de conocimiento, por ejemplo, sobre la efectividad de algunos procedimientos terapéuticos que no "conversan" con resultados de otras investigaciones ni contribuyen a comprender el fenómeno del cambio psicoterapéutico en términos más generales, y a la construcción de teorías de mayor envergadura. 
Asimismo, cuando la investigación se distancia de la práctica clínica, pierde su pertinencia y potencial impacto, al dejar de nutrirse de las preguntas que son relevantes para quienes ejercen las intervenciones y caer en el encapsulamiento dentro de los ámbitos científicos. En respuesta a esto, y a fin de disminuir la brecha entre investigación en psicoterapia y ejercicio clínico, en los últimos años ha surgido un enfoque denominado investigación orientada a la práctica (Castonguay, 2017) que involucra profesionales clínicos en el diseño y ejecución de los estudios, con el objetivo de dar mayor protagonismo a su pertinencia profesional (Castonguay \& Muran, 2015).

Por último, cabe preguntarse en qué derivaría el ejercicio clínico si no interactuara con la teoría, por una parte, y la investigación, por otra. Está bien establecido que apoyarse en una teoría o enfoque aporta a la efectividad del clínico (Frank, 1984). La teoría le da coherencia a su quehacer, permite la planificación de la terapia y, hasta cierto punto, la evaluación de sus resultados; además, le provee de herramientas terapéuticas específicas, que son el vehículo de su acción transformadora. No obstante, la evaluación del propio trabajo tiene importantes sesgos. Los seres humanos, si bien podemos estar convencidos de nuestra "mente científica", dado que formulamos hipótesis que contrastamos a través de nuestras prácticas (Kelly, 1955), en la práctica sufrimos de "sesgo positivo", vale decir, percibimos más la evidencia que confirma nuestras hipótesis, que la que las desconfirma (Alicke, 1985). Por esta razón, el clínico no se debe bastar a sí mismo como investigador, sino que requiere de agentes externos que puedan someter a prueba la efectividad de su quehacer.

Por otra parte, dado que la psicoterapia y las intervenciones en salud dependen de agentes gubernamentales y financiadores, que crecientemente se interesan por la efectividad (y costo-efectividad) de los procedimientos que financian, la investigación en psicoterapia tiene un importante rol en la validación del quehacer clínico. Este ejercicio favorecerá la inclusión de procedimientos psicoterapéuticos empíricamente validados e informados en los planes y políticas de salud mental de los países, lo cual es una tarea aún no terminada en muchos países del subcontinente latinoamericano.

El presente número especial de la revista CES Psicología editada por la Facultad de Psicología de la Universidad CES (Colombia) aporta en este sentido. Cumple con el objetivo de fortalecer el diálogo entre teoría, investigación y práctica clínica, y es -en sí mismo- un vehículo para la visibilización del quehacer investigativo latinoamericano, dentro de la región, y en otras partes del mundo.

Teniendo en cuenta lo anterior, el número especial incluye trabajos de reflexión teórica; otros focalizados en instrumentos de investigación, incluyendo su validación, adaptación y descripción; resultados de investigación psicoterapéutica con adultos, con niños y adolescentes, tanto de micro-proceso como de macroproceso; y de evaluación y desarrollo de intervenciones.

Los trabajos representan un abanico de investigación en psicoterapia de América Latina en términos temáticos, metodológicos y territoriales. De esta manera, autores de Argentina, Brasil, Chile, Colombia, Ecuador y Perú, con el aporte de investigadores de Alemania, siendo la mayoría de ellos investigadores del capítulo latinoamericano de la Society for Psychotherapy Research (SPR), aportan múltiples reflexiones y datos empíricos de alta pertinencia clínica y que, a su vez, amplían las preguntas y desafíos que tenemos como región en este campo. Finalmente, este número también constituye 
una preparación para el futuro Congreso Latinoamericano de Investigación en Psicoterapia, que se llevará a cabo en la ciudad de Medellín (Colombia).

\section{Referencias}

Alicke, M.D. (1985). Global self-evaluation as determined by the desirability and controllability of trait adjectives. Journal of Personality and Social Psychology, 49, 1621-1630. https://doi.org/10.1037/0022-3514.49.6.1621

Angus, L. E., \& Kagan, F. (2013). Assessing client self-narrative change in emotion-focused therapy of depression: an intensive single case analysis. Psychotherapy, 50(4), 525-34. https://doi.org/10.1037/a0033358

Bastine, R., Fiedler, P., \& Kommer, D. (1989). Was ist psychotherapeutisch an der Psychotherapie? Versuch einer Bestandsaufnahme und Systematisierung der psychotherapeutischen Prozeßforschung. Zeitschrift für Klinische Psychologie, 18, 3-22.

Bordin, E.S. (1976). The generalization of the psychoanalytic concept of the working alliance. Psychotherapy: Theory, Research and Practice, 16, 252-260.

Braakmann, D. (2014). Historical Paths in Psychotherapy Research. In Gelo O., Pritz A., \& Rieken B. (eds) Psychotherapy Research (39-65). Viena: Springer-Verlag Publishing. https://doi.org/10.1007/978-3-7091-1382-0 3

Castonguay, L. (2017). Practice-Oriented Research in Psychotherapy. https://doi. org/10.4324/9781315644271

Castonguay, L. G., \& Muran, J. C. (2015). Fostering collaboration between researchers and clinicians through building practice oriented research: An introduction. Psychotherapy Research, 25, 1-5. https://doi.org/10.1080/10503307.2014.966348

Castonguay, L. G., Youn, S. J., Xiao, H., Muran, J. C., \& Barber, J. P. (2014). Building clinicians-researchers partnerships: Lessons from diverse natural settings and practice-oriented initiatives. Psychotherapy Research, 25(1), 166-184. https:// doi.org/10.1080/10503307.2014.973923

Comer, J., \& Kendall, P. (2013). Methodology, Design, and Evaluation in Psychotherapy Research. In M.J. Lambert (ed). Bergin and Garfield's Handbook of Psychotherapy and Behavior Change, $(21-48)$. 6th ed. Hoboken, NJ: Wiley.

De la Parra, G., Gómez-Barris, E., Zúñiga, A. K., Dagnino, P., \& Valdés, C. (2018). Del "diván" al policlínico: Un modelo de psicoterapia para instituciones. Aprendiendo de la experiencia (empírica) [From the "couch" to the outpatient clinic: A model of psychotherapy for institutions. Learning from (empirical) experience]. Revista Argentina de Clínica Psicológica. 27(2), 182-202.

Elliott, R. (1991). Five Dimensions of Therapy Process. Psychotherapy Research, 1(2), 92-103, http://doi.org/10.1080/10503309112331335521

Elliott, R. (1985). Helpful and nonhelpful events in brief counseling interviews: An empirical taxonomy. Journal of Counseling Psychology, 32(3), 307-322. https:// doi.org/10.1037/0022-0167.32.3.307

Fernández-Álvarez, H., \& Castonguay, L. G. (2018). Investigación orientada por la práctica: Avances en colaboraciones entre clínicos e investigadores. Introducción. Revista Argentina de Clínica Psicológica, 27, 107-110. https://doi. org/10.24205/03276716.2018.1069

Fernández-Álvarez, J., Prado-Abril, J., Sánchez-Reales, S., Molinari, G., Martín Gómez Penedo, J., ... Jeong Youn, S. (2020). La brecha entre la investigación y la práctica clínica: Hacia la integración de la psicoterapia. Papeles Del Psicólogo, 41(2). https://doi.org/10.23923/pap.psicol2020.2932

Frank, J. D. (1984). Persuasion and Healing. The British Journal of Psychiatry, 145, $207-$ 211. https://doi.org/10.1192/S0007125000205084 
Gelo, O., Pritz, A., \& Rieken, B. (Eds.) (2015). Psychotherapy Research. Foundations, Process, and Outcome. Viena: Springer-Verlag Publishing. https://doi. org/10.1007/978-3-7091-1382-0

Gennaro, A., Al-Radaideh, A., Gelo, O., Manzo, S., Nitti, M., Auletta, A., \& Salvatore, S. (2010). Modelling the psychotherapy process as a meaning making dynamics. The Two Stage Semiotic Model (TSSM) and the Discourse Flow Analyzer (DFA). In S. Salvatore, J. Valsiner, J. Travers Simon, \& A. Gennaro (Eds.), Yearbook of idiographic science 2009-Volume 2 (pp. 131-170). Roma: Firera Publishing Group.

Hardy, G. E., \& Llewelyn, S. (2015). Introduction to psychotherapy process research. In O. C. G. Gelo, A. Pritz, \& B. Rieken (Eds.), Psychotherapy research: Foundations, process, and outcome (p. 183-194). Viena: Springer-Verlag Publishing. https:// doi.org/10.1007/978-3-7091-1382-0 9

Herrera Salinas, P., Fernández González, O., Krause, M., Vilches Álvarez, O., Valdés, N., \& Dagnino, P. (2009). Revisión teórica y metodológica de las dificultades en Psicoterapia: Propuesta de un Modelo Ordenador. Terapia Psicológica, 27, 169-179.

Holmqvist Larsson, M., Falkenström, F., Andersson, G., \& Holmqvist, R. (2018) Alliance ruptures and repairs in psychotherapy in primary care, Psychotherapy Research, 28(1), 123-136, https://doi.org/10.1080/10503307.2016.1174345

Horvath, A. O., \& Greenberg, L. S. (1986). The development of the Working Alliance Inventory. In L. S. Greenberg, \& W. Pinsoff (Eds.), The psychotherapeutic process: A research handbook (pp. 529-556). New York: Guildford Press.

Jaramillo, J. C., Espinosa-Duque, D., Ocampo, D., Arango, A., Arévalo, M., García, L., ... Sandoval C. (2018). Caracterización de la atención psicoterapéutica en la IPS CES Sabaneta, Colombia, 2014-2015. CES Psicología, 11(2), 97-110. https://dx. doi.org/10.21615/cesp.11.2.9

Jiménez, J. (1999). ¿Investigación en psicoterapia? Claro que sí. Pero, ¿A quién le interesa?. Revista de Psicología, 8(1), 105-116. https://doi.org/10.5354/0719$\underline{0581.1999 .17178}$

Kelly, G. A. (1955). The Psychology of Personal Constructs. New York Norton.

Krause, M. (2011a). Psicoterapia y Cambio. Una mirada desde la subjetividad. Santiago de Chile: Ediciones Universidad Católica.

Krause, M. (2011b). La Psicoterapia: ¿Oficio sin Ciencia y Ciencia sin Oficio?. Revista Colombiana de Psicología, 20(1), 89-98.

Krause, M., \& Altimir, C. (2016) Introduction: current developments in psychotherapy process research / Introducción: desarrollos actuales en la investigación del proceso psicoterapéutico, Estudios de Psicología, 37, 2-3, 201-225, https://doi.or $\mathrm{g} / 10.1080 / 02109395.2016 .1227574$

Krause, M., Altimir, C., Pérez, J.C., \& De la Parra, G. (2015). Generic Change Indicators in therapeutic processes with different outcomes. Psychotherapy Research, 25(5), 533-545.

Krause, M., Espinosa, H. D., Tomicic, A., Córdoba, A. C., \& Vásquez, D. (2018). Psychotherapy for depression from the point of view of economically disadvantaged individuals in Chile and Colombia. Counselling and Psychotherapy Research 18(2), 178-189. https://doi.org/10.1002/capr.12171

Llewelyn, S., \& Hardy, G. (2001). Process research in understanding and applying psychological therapies. The British journal of clinical psychology / the British Psychological Society. 40.1-21. https://doi.org/10.1348/014466501163436

Matos, M., Santos, A., Gonçalves, M., \& Martins, C. (2009). Innovative moments and change in narrative therapy: Psychotherapy Research, 19(1), 68-80, https://doi. org/10.1080/10503300802430657 
Meyerhoff, J., \& Rohan, K.J. (2016). Treatment expectations for cognitive-behavioral therapy and light therapy for seasonal affective disorder: Change across treatment and relation to outcome. Journal of Consulting and Clinical Psychology, 84(10), 898-906.

Norcross, J. C., \& Lambert, M. J. (2011). Psychotherapy relationships that work II. Psychotherapy, 48(1), 4-8. https://doi.org/10.1037/a0022180

Orlinsky, D. (1994). Process and outcome in psychotherapy. En Bergin AE, Garfield SL (eds) Handbook of psychotherapy and behavior change, Nueva York: John Wiley.

Prochaska, J. O., \& Norcross, J. C. (2002). Stages of change. In J. C. Norcross (Ed.), Psychotherapy relationships that work: Therapist contributions and responsiveness to patients (pp. 303-313). New York, NY: Oxford University Press.

Rogers, C. R. (1957). The necessary and sufficient conditions of therapeutic personality change. Journal of Consulting Psychology, 21(2), 95-103. https://doi. org/10.1037/h0045357.

Safran, J., Muran, C. (2000). Resolving therapeutic alliance ruptures: Diversity and integration. Journal of Clinical Psychology, vol 56, n.2. https://doi.org//10.1002/ (SICI)1097-4679(200002)56:2<233::AID-JCLP9>3.0.C0;2-3

Safran, J. D., Muran, J. C., \& Eubanks-Carter, C. (2011). Repairing alliance ruptures. Psychotherapy, 48(1), 80-87. https://doi.org/10.1037/a0022140

Stiles, W. B. (2001). Assimilation of problematic experiences. Psychotherapy, 38, 462 $-465$.

Stiles, W. B. (2002). Assimilation of problematic experiences. In J. C. Norcross (Ed.), Psychotherapy relationships that work: Therapist contributions and responsiveness to patients (pp. 357-365). New York, NY: Oxford University Press.

Timulák, L., \& Lietaer, G. (2001). Moments of empowerment: A qualitative analysis of positively experienced episodes in brief person-centred counselling, Counselling and Psychotherapy Research: Linking research with practice, 1(1), 62-73. http:// dx.doi.org/10.1080/14733140112331385268

Tomicic, A., Martínez, C., \& Krause, M. (2015). The sound of change: A study of the psychotherapeutic process embodied in vocal expression. Laura Rice ideas revisited. Psychotherapy Research, 25(2), 263-276. https://doi. org10.1080/10503307.2014.892647

Valdés, N., Gómez, D., \& Reinel, M. (2017). Análisis de los momentos de ruptura y reparación de la alianza terapéutica: estudio de caso de una adolescente diagnosticada con difusión de identidad" ¿Qué es lo que Cambia en la Psicoterapia? La perspectiva de Pacientes Adolescentes con Síntomas Depresivos, sus Terapeutas y Observadores Externos. Psykhe ,27, 2, 1-20, https://doi.org/10.7764/ psykhe.27.2.1136

Vöhringer, C., Pérez, J. C., Martínez, M., Altimir, C., Dagnino, P., Suarez, N., \& Krause, M. (2013). Working Alliance Inventory versión observacional: Traducción, adaptación y validación al castellano. Terapia Psicológica, 31(3), 301-311. http://dx.doi. org/10.4067/S0718-48082013000300005

Von Bergen, A., \& Krause, M. (2016). Disonancias, armonías y diálogos entre la investigación y la clínica. Psiquiatría y Saud Mental, 23(1/2), 5-15.

Wampold, B. E. (2013). The Great Psychotherapy Debate. Models, Methods, and Findings. (1st Edition). https://doi.org/10.4324/9780203893340

Watkins, C.E. (1997). Reflections on Contemporary Psychotherapy Practice, Research, and Training. Journal of Contemporary Psychotherapy. 27, 5-22. 